\title{
Monitoring Nanoscale Deformations in a Drawn Polymer Melt with Single-Molecule Fluorescence Polarization Microscopy
}

\author{
Supporting Information \\ Stefan Krause, * Martin Neumann, Melanie Fröbe, Robert Magerle, * \\ and Christian von Borczyskowski
}

Fakultät für Naturwissenschaften, Technische Universität Chemnitz, 09107 Chemnitz, Germany 


\section{Exclusion of Birefringence}

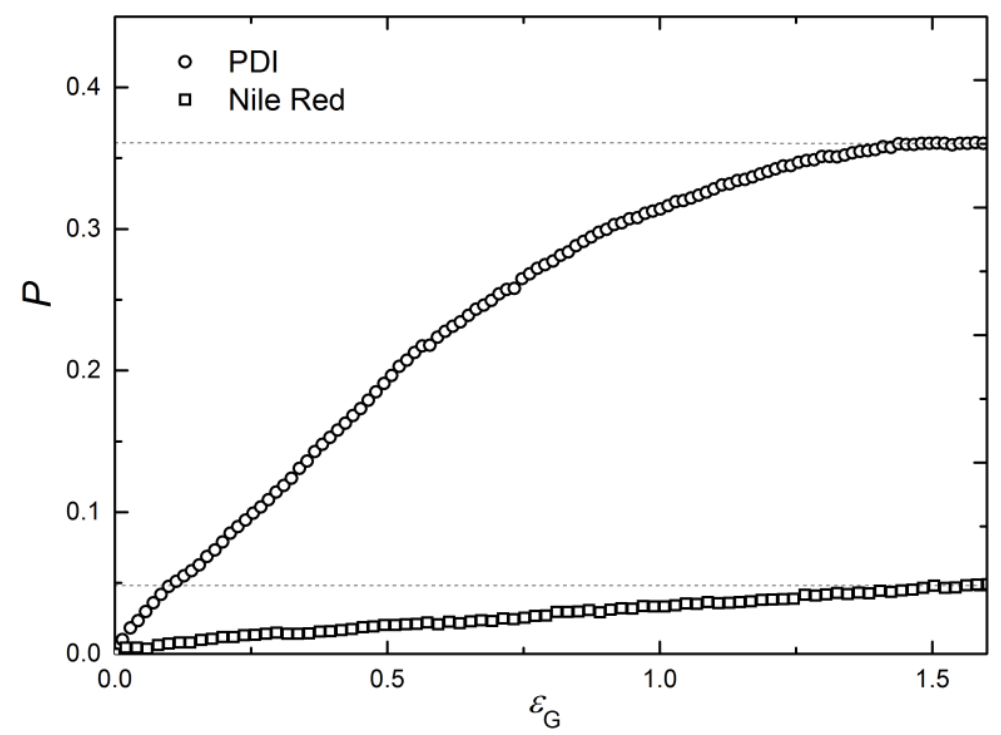

Figure S1. Fluorescence polarization of PDI and Nile red as a function of global elongation $\varepsilon_{\mathrm{G}}$. The data were measured at $285 \mathrm{~K}$ at the ensemble level, and the elongation rate was $\dot{\varepsilon}_{\mathrm{G}}$ $=0.014 \mathrm{~s}^{-1}$. The horizontal dashed lines indicate the maximal achieved polarization. Nile red molecules are almost spherical and orient much less than PDI molecules. This excludes birefringence as a dominant polarizing process, as it would not depend on the type of molecule. 


\section{Temporal evolution of the orientation angles}

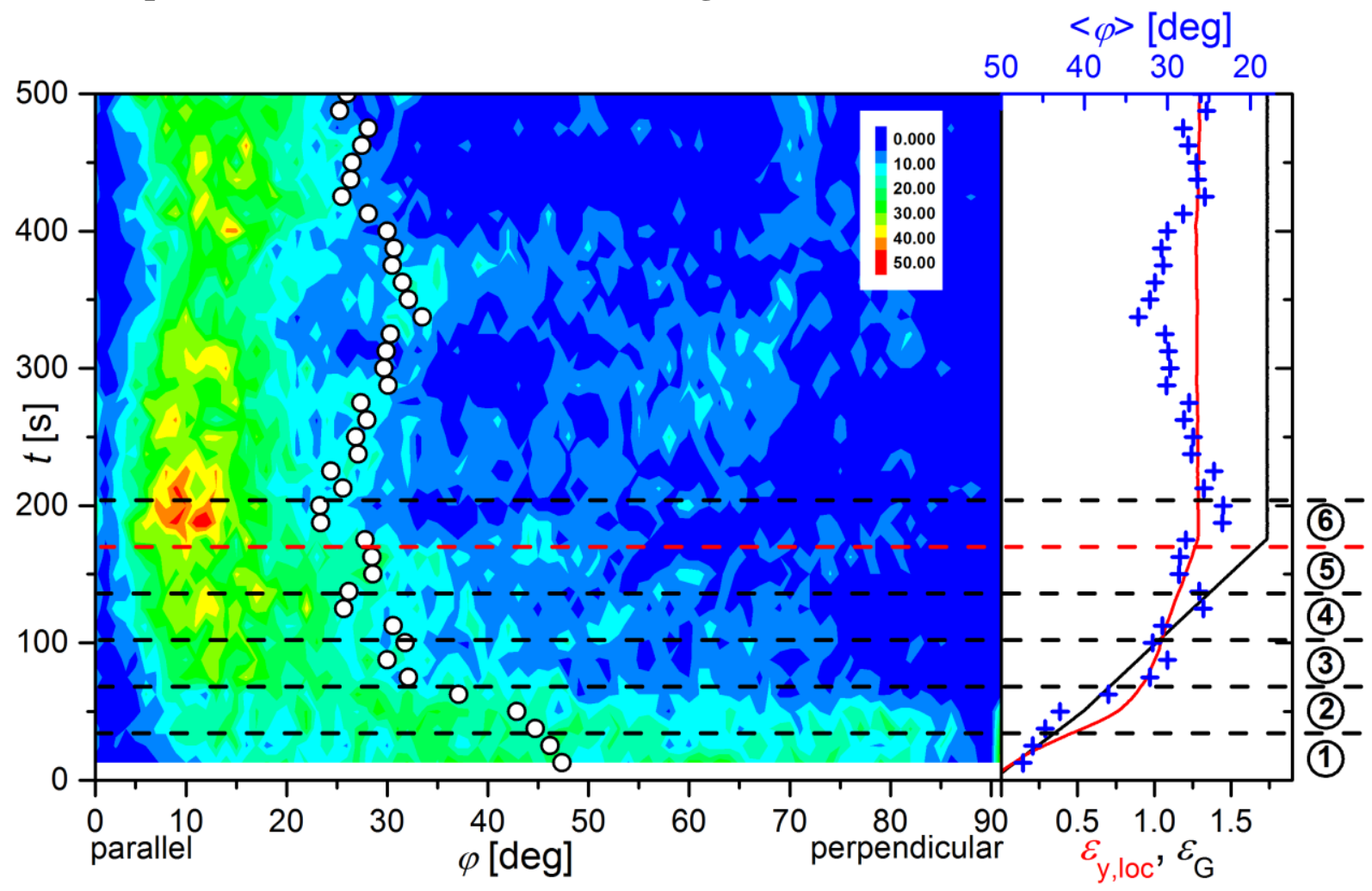

Figure S2. Left side: Time-dependent evolution of the distribution of the in-plane orientation angles $\varphi$ of single PDI molecules in PMA at $285 \mathrm{~K}$. The contour color plot consists of 40 histograms, each averaged over $12.5 \mathrm{~s}$. The inset shows the color encoding, while black and white circles mark the average orientation angles $\langle\varphi\rangle$. The experiment starts with an equal distribution of orientations at zero elongation at $t=0 \mathrm{~s}$. The local elongation increases to $\varepsilon_{\mathrm{y}, \mathrm{loc}}=1.3$ within $170 \mathrm{~s}$. The end of the elongation period is indicated by the red dashed horizontal line. Black dashed lines divide the elongation period into six equal intervals which are numbered at the right side and correspond to the histograms shown in Figure 3. Right side: Temporal evolution of the global $\varepsilon_{\mathrm{G}}$ and the local $\varepsilon_{\mathrm{y}, \mathrm{loc}}$ elongation (black and red lines, respectively) of the film as well as the average orientation angle $\langle\varphi\rangle$ (blue crosses, same data as shown on the left side). 


\section{Simulation Details}

We start with an isotropic distribution of orientation angles, which is expected for dye molecules in a relaxed polymer film at $\varepsilon_{\mathrm{y}, \mathrm{loc}}=0$. The initial in-plane angles $\varphi_{0}$ are chosen to be uniformly distributed between $0^{\circ}$ and $360^{\circ}$, while the initial out-of-plane angles $\theta_{0}$ are distributed proportional to a cosine function between $0^{\circ}$ and $180^{\circ}$. This results in an isotropic distribution of molecular orientation vectors as shown in Figure S3a. The dye molecules are modeled as rod-like objects embedded in a uniaxially elongated thin film with conserved volume (Figure 5). Upon uniaxial elongation $\varepsilon_{\mathrm{y} \text {,loc }}$ in the y-direction, the initial volume element of size $b_{0} \cdot d_{0} \cdot l_{0}$ is deformed. Its length increases by a factor of $\left(1+\varepsilon_{y, l o c}\right)$, and the film thickness decreases by a factor of $\left(\varepsilon_{\mathrm{y}, \mathrm{loc}}+1\right)^{-1}$. The width $b_{0}$ of the volume element does not change. The resulting orientation angles of the diagonal are given by

$$
\varphi_{\mathrm{S}}=\arctan \left(\frac{\tan \varphi_{0}}{\varepsilon_{\mathrm{y}, \mathrm{loc}}+1}\right)
$$

and

$$
\theta_{\mathrm{S}}=\arctan \left(\frac{\tan \theta_{0} \sin \varphi_{0}\left(\varepsilon_{\mathrm{y}, \text { loc }}+1\right)}{\sin \left[\arctan \left(\frac{\tan \varphi_{0}}{\varepsilon_{\mathrm{y}, \text { loc }}+1}\right)\right]}\right) .
$$

The distribution of the orientation angles calculated according to Eqs. (1) and (2) for $\varepsilon_{\mathrm{y}, \mathrm{loc}}=1.3$ is shown in Figure $\mathrm{S} 3 \mathrm{~b}$. 
a) $\varepsilon_{y, \text { loc }}=0$
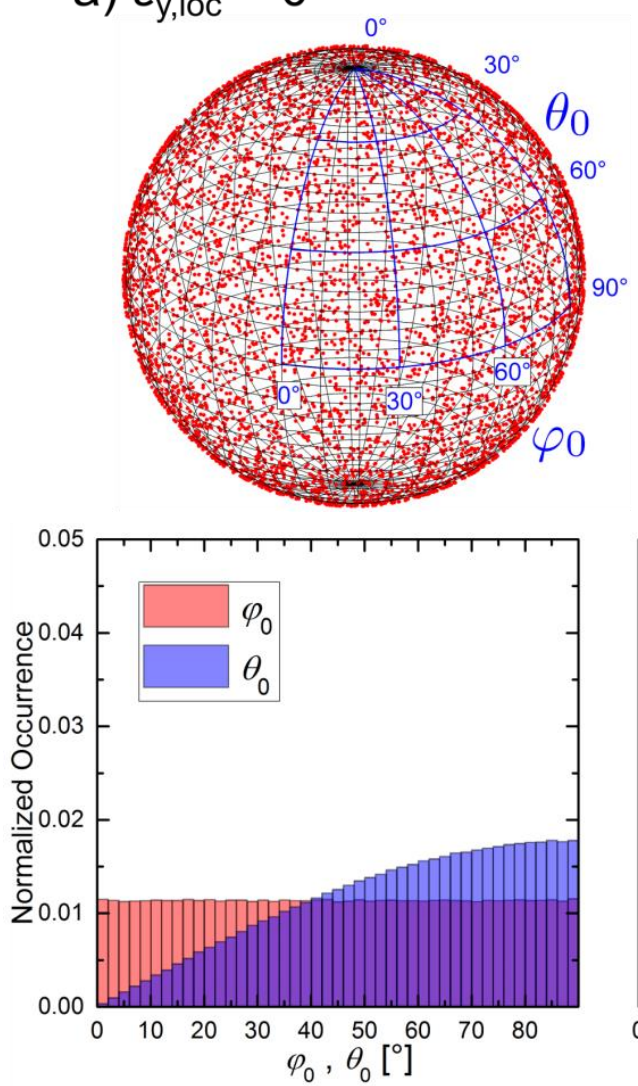

b) $\varepsilon_{y, l o c}=1.3_{0^{\circ}}$
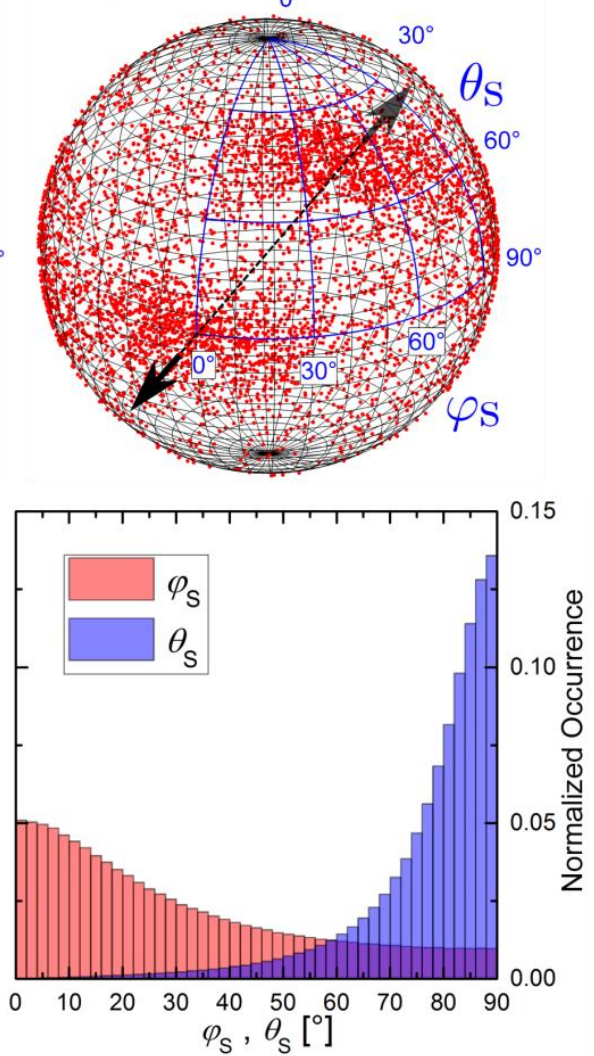

Figure S3. (a) Spherical plot of orientation vectors (top) for an initially isotropic distribution (bottom) at $\varepsilon_{\mathrm{y}, \mathrm{loc}}=0$ and (b) for an anisotropic distribution at $\varepsilon_{\mathrm{y}, \mathrm{loc}}=1.3$. The spherical plots are also shown in Figure 5. Each red spot represents a pair of in- and out-of-plane angles $\varphi_{0}, \theta_{0}$ and $\varphi_{\mathrm{S}}, \theta_{\mathrm{S}}$. The distribution of orientation angles are calculated according to Eqs. (1) and (2).

The expected fluorescence intensity $\hat{I}^{\mathrm{P}}(\varphi, \theta)$ (parallel to the elongation direction) and $\hat{I}^{S}(\varphi, \theta)$ (perpendicular to the elongation direction) for a molecule with orientation angles $(\varphi, \theta)$ are

$$
\begin{aligned}
& \hat{I}^{\mathrm{P}}(\varphi, \theta)=I_{\mathrm{tot}} \cdot\left\{A+B \cdot \sin ^{2} \theta+C \cdot \sin ^{2} \theta \cos (2 \varphi)\right\} \cdot \cos ^{2} \theta \\
& \hat{I}^{\mathrm{S}}(\varphi, \theta)=I_{\mathrm{tot}} \cdot\left\{A+B \cdot \sin ^{2} \theta-C \cdot \sin ^{2} \theta \cos (2 \varphi)\right\} \cdot \cos ^{2} \theta .
\end{aligned}
$$


The formulas take the numerical aperture of the microscope objective and the decreased probability for exciting molecules oriented out of the film plane into account. ${ }^{1-3} I_{\mathrm{tot}}$ is the total emitted intensity and $A, B$ and $C$ are constants determined by the numerical aperture of the microscope objective or the aperture angle $\alpha$ :

$$
\begin{aligned}
& A=\frac{1}{6}-\frac{1}{4} \cos \alpha+\frac{1}{12} \cos ^{3} \alpha \\
& B=\frac{1}{8} \cos \alpha-\frac{1}{8} \cos ^{3} \alpha \\
& C=\frac{7}{48}-\frac{1}{16} \cos \alpha-\frac{1}{16} \cos ^{2} \alpha-\frac{1}{48} \cos ^{3} \alpha
\end{aligned}
$$

On the CCD camera, the image of a single molecule is a diffraction-limited spot with the number of counts $N_{i j}^{\mathrm{P}}$ and $N_{i j}^{\mathrm{S}}$ at the pixel $(\mathrm{i}, \mathrm{j})$ in the p- and s-polarization channels, respectively, given by

$$
\begin{aligned}
& N_{i j}^{\mathrm{P}}(\varphi, \theta)=\hat{I}^{\mathrm{P}}(\varphi, \theta) \cdot \exp \left(-\frac{\left(i-i_{C}\right)^{2}+\left(j-j_{C}\right)^{2}}{2 \varsigma^{2}}\right) \\
& N_{i j}^{\mathrm{S}}(\varphi, \theta)=\hat{I}^{\mathrm{S}}(\varphi, \theta) \cdot \exp \left(-\frac{\left(i-i_{C}\right)^{2}+\left(j-j_{C}\right)^{2}}{2 \varsigma^{2}}\right),
\end{aligned}
$$

where $\left(i_{C}, j_{C}\right)$ describe the center of the spot positions. The variance $\varsigma^{2}$ is set to 1.5 pixels, corresponding to the variance of the measured spot size. For simulating an image sequence corresponding to the measured sequence of CCD images, we assume $n_{0}=50$ spots at random positions within the observation area for $\varepsilon_{y, l o c}=0$. This number corresponds to the initial number of observed spots in the experiment. The simulated signal in the p-polarization channel is a sum of $n$ diffraction-limited spots and the background $B_{i j}\left(\varepsilon_{\mathrm{y}, \mathrm{loc}}\right)$ 


$$
M_{i j}^{\mathrm{P}}\left(\varepsilon_{\mathrm{y}, \mathrm{loc}}\right)=\sum_{k=1}^{n} N_{i j, k}^{\mathrm{P}}(\varphi, \theta)+B_{i j}\left(\varepsilon_{\mathrm{y}, \mathrm{loc}}\right)
$$

For each molecule $k$, we start with a random initial orientation $\left(\varphi_{0}, \theta_{0}\right)$ which changes as a function of $\varepsilon_{y, l o c}$, given by Eqs. (1) and (2). For each frame, the number of molecules within the observed area is given by $n\left(\varepsilon_{\mathrm{y}, \text { loc }}\right)=n_{0} /\left(1+\varepsilon_{\mathrm{y}, \mathrm{loc}}\right)$. The background $B_{i j}\left(\varepsilon_{\mathrm{y}, \mathrm{loc}}\right)$ is set to a distribution of random values which obey a normal distribution with an expectation value $b_{\mathrm{C}}$ and a variance $\sigma^{2}$. The values for $b_{\mathrm{C}}$ and $\sigma^{2}$ were determined from the measured sequence of singlemolecule images for each frame (see Figure S4).

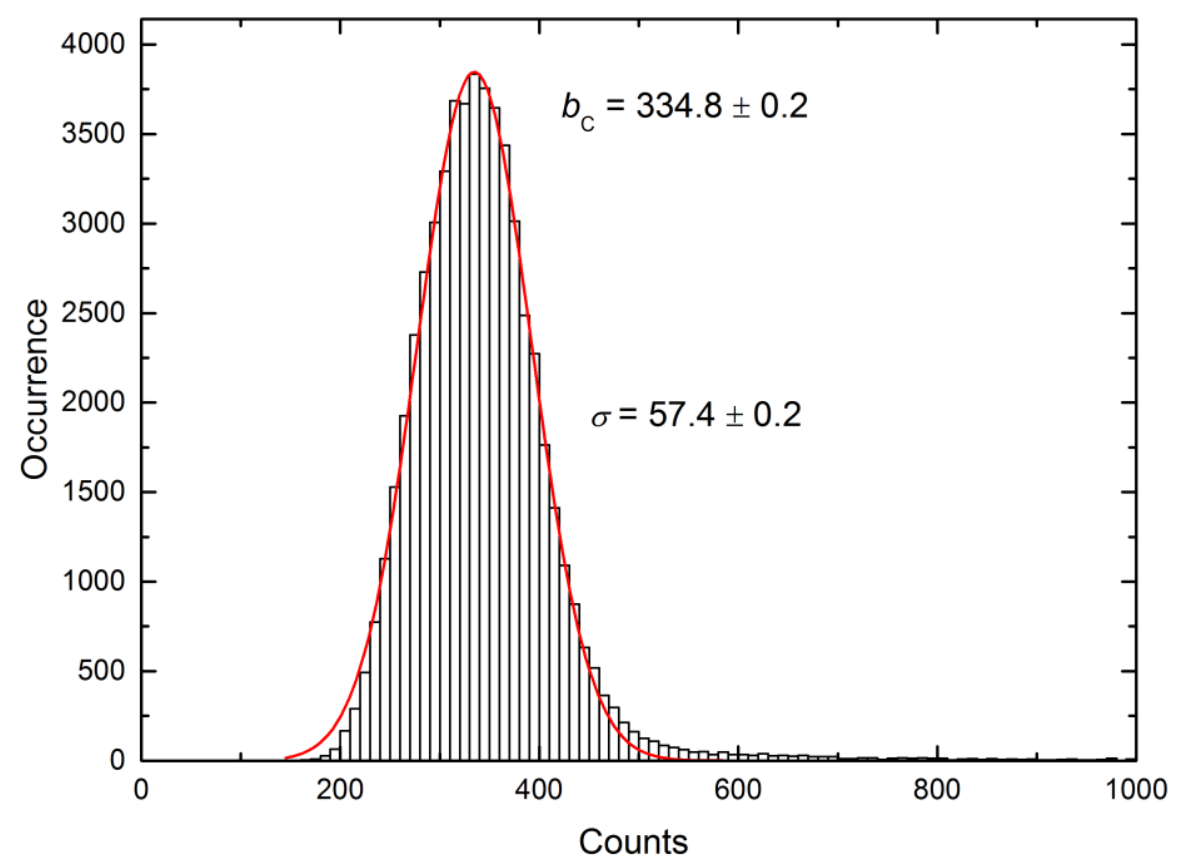

Figure S4. Histogram of pixel values of a $322 \times 176$ large single-molecule image containing the s-polarization channel and p-polarization channel (see Figure S5a). The small asymmetry of the distribution is due to the fluorescence of the single molecules. The distribution was fitted by a Gaussian function to determine the expectation value $x_{\mathrm{C}}$ and the variance of the noise $\sigma^{2}$. An example of the resulting simulated image for $\varepsilon_{\mathrm{y}, \mathrm{loc}}=1.3$ is shown in Figure S5, where the measured single-molecule image for $\varepsilon_{\mathrm{y}, \mathrm{loc}}=1.3$ is shown for comparison. 


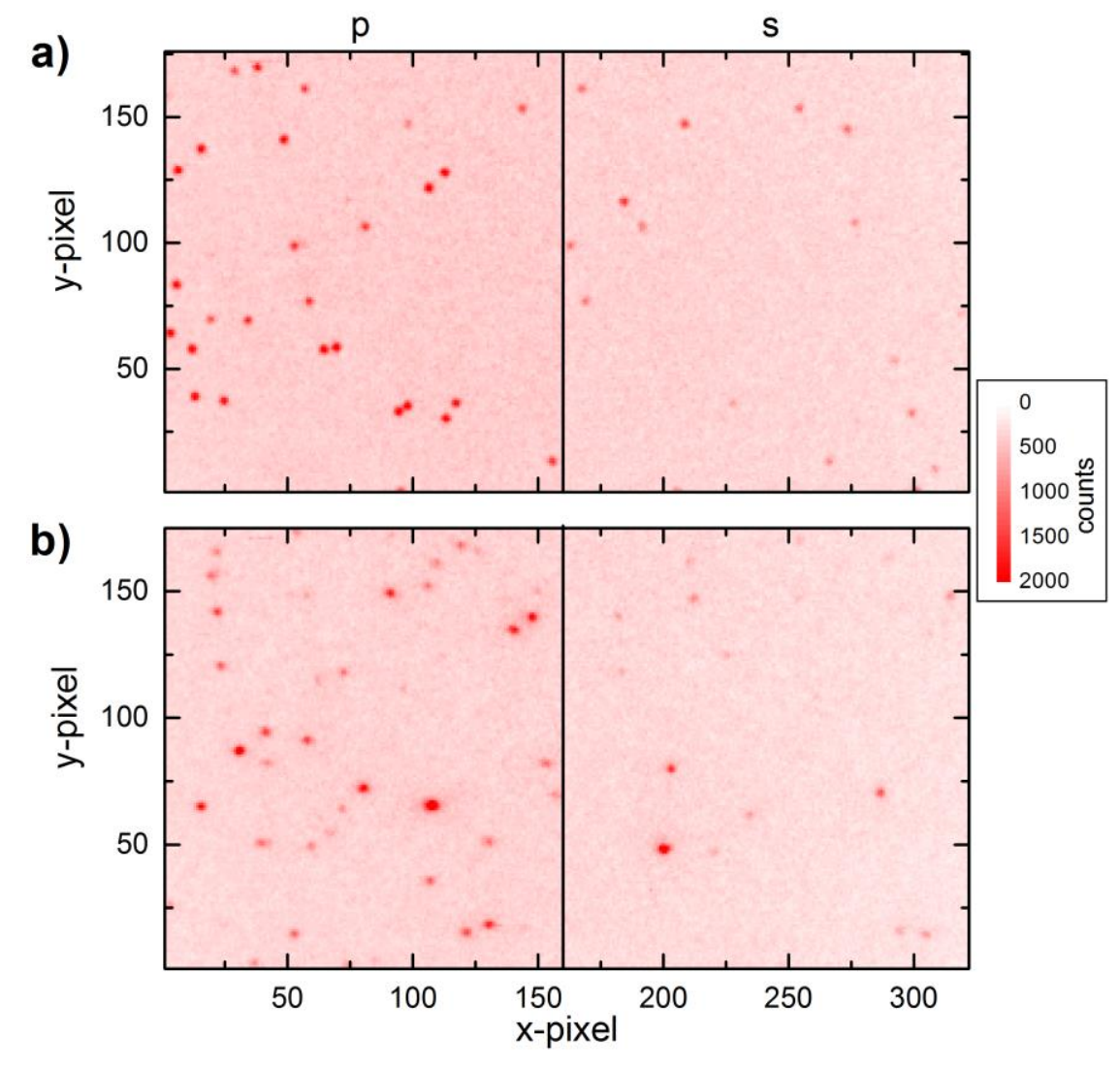

Figure S5. Examples of the simulated (a) and experimental (b) SM images for a 1.3-timeselongated polymer film. The left side shows the fluorescence signal of the detection channel with polarization parallel to the direction of elongation; the right side shows the channel with perpendicular polarization. 


\section{Number of Molecules and Error for In-Plane Angle Determination}
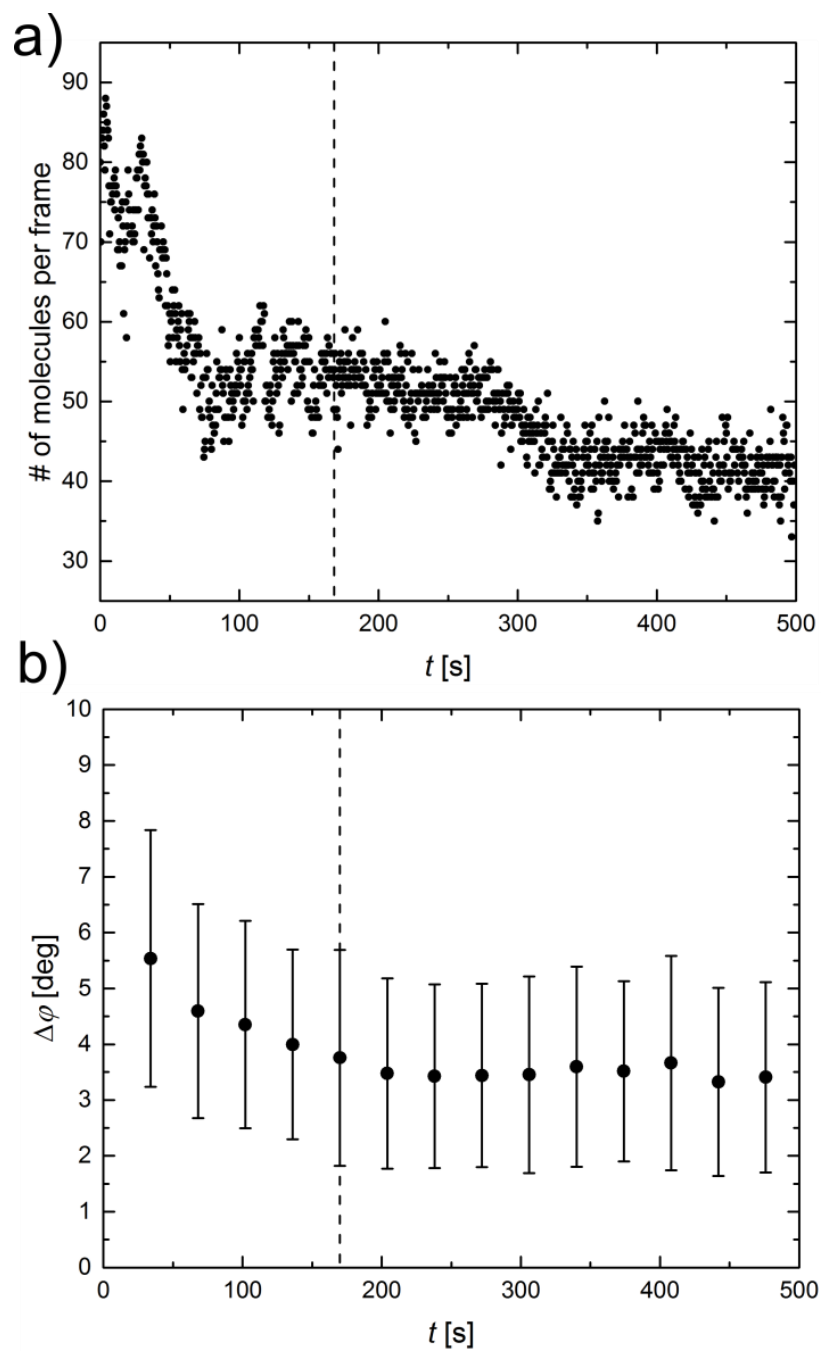

Figure S6. a) Number of PDI molecules detected in each frame as a function of time. b) Error for the determination of the in-plane orientation angle $\varphi$, averaged over all molecules within 68 consecutive frames. The error bars indicate the spread of the error. 


\section{Comparison of Relaxation Times for Polymethyl Acrylate Melts}

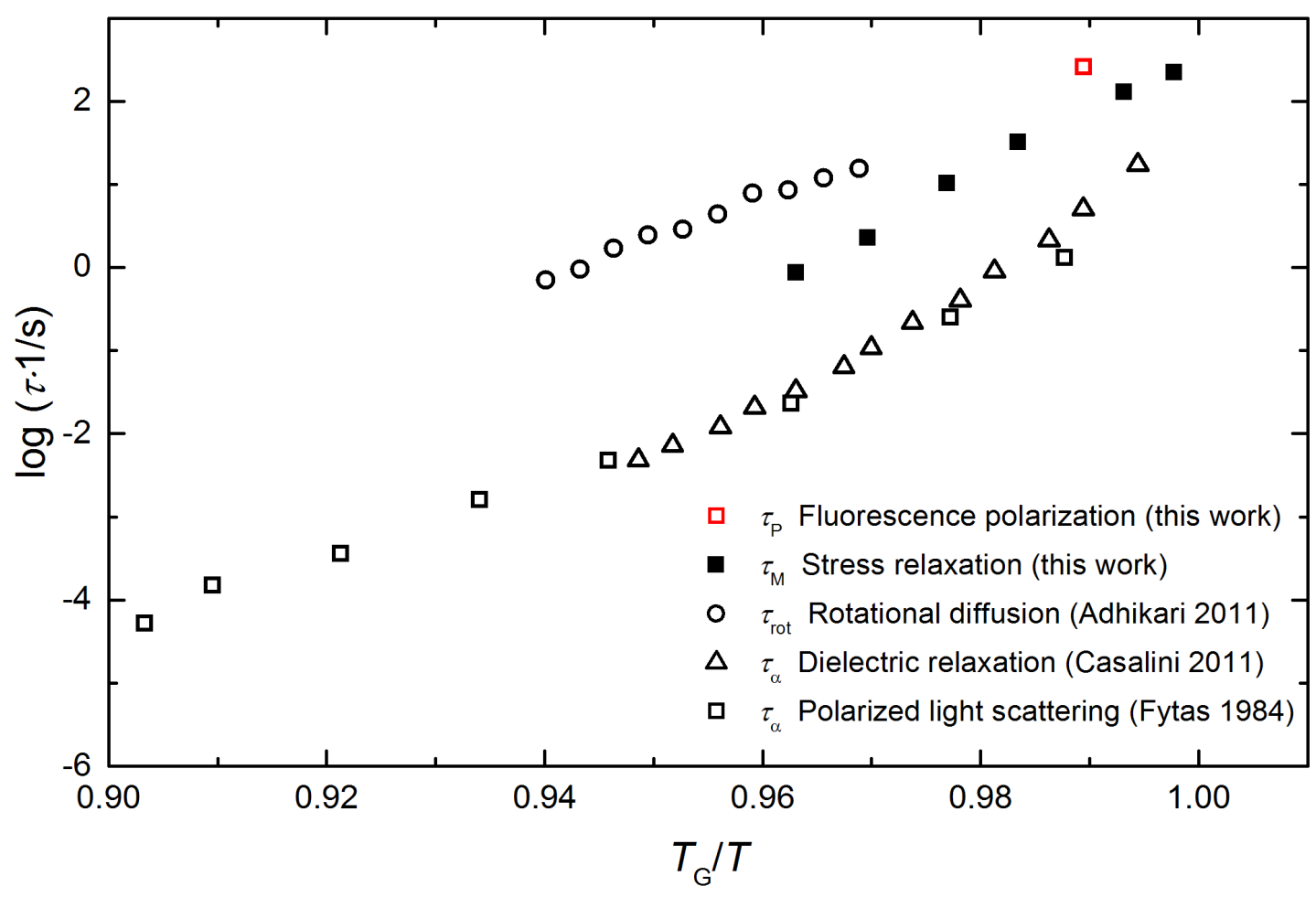

Figure S7. Logarithm of the relaxation time of the fluorescence polarization (red square) and the stress relaxation (black solid squares), the mean correlation time characterizing rotational diffusion (open circles), ${ }^{3}$ the mean relaxation time of the $\alpha$-relaxation process measured with dielectric relaxation spectroscopy (open triangles), ${ }^{4}$ and photon correlation polarized light scattering (open squares), ${ }^{5}$ plotted as a function of $T_{\mathrm{G}} / T$. 


\section{References}

(1) Fourkas, J. T., Rapid Determination of the Three-Dimensional Orientation of Single Molecules. Opt. Lett. 2001, 26, 211-213.

(2) Hohlbein, J.; Hübner, C. G., Three-Dimensional Orientation Determination of the Emission Dipoles of Single Molecules: The Shot-Noise Limit. J. Chem. Phys. 2008, 129, 094703.

(3) Adhikari, S.; Selmke, M.; Cichos, F., Temperature Dependent Single Molecule Rotational Dynamics in PMA. Phys. Chem. Chem. Phys. 2011, 13, 1849-1856.

(4) Casalini, R.; Fragiadakis, D.; Roland, C. M., Relaxation Dynamics of Poly(Methyl Acrylate) at Elevated Pressure. Macromolecules 2011, 44, 6928-6934.

(5) Fytas, G.; Patkowski, A.; Meier, G.; Dorfmüller, T., A High Pressure Photon Correlation Study of Bulk Poly (Methylacrylate). Comparison with Relaxation Processes in Poly (Ethylacrylate) and Related Polymethacrylates. J. Chem. Phys. 1984, 80, 2214-2220. 